\title{
Brainstem tumors in children: a review
}

\author{
Matheus Fernando Manzolli Ballestero', Luciano Lopes Furlanetti ${ }^{2}$, Ricardo Santos de \\ Oliveira $^{3}$
}

Received: 01 December 2020 / Published: 15 January 2021

\begin{abstract}
The brainstem is defined as the portion of the neural axis between the diencephalon and the cervical spinal cord. Brainstem gliomas (BSGs) can occur anywhere within this region and account for $10 \%$ to $15 \%$ of primary pediatric intracranial tumors. The development of neuroimaging allied to the refinement of surgical tools, such as improved magnification and visualization of the surgical field with more powerful surgical microscopes and sources of light, followed by the introduction of ultrasonic aspirator, anesthetic and intraoperative neurophysiological monitoring provided the neurosurgeon with conditions for a much safer surgical management of these tumors. This article reviews the current state of knowledge with regard to tumors arising in the brain stem in children, the therapeutic options available and provides recommendations with regard to management.
\end{abstract}

Key words: Tumors, brainstem, review, glioma

\section{Introduction}

The brainstem is defined as the portion of the neural axis between the diencephalon and the cervical spinal cord. Brainstem gliomas (BSGs) can occur

${ }^{1}$ Department of Medicine, Federal University of São Carlos, Brazil

2Department of Basic and Clinical Neuroscience, King's College London, London, UK

${ }^{3}$ Division of Neurosurgery, Department of Surgery and Anatomy, University of São Paulo, Ribeirao Preto, Brazil

To whom correspondence should be addressed: Matheus Ballestero, MD, MSc [E-mail: ballestero@gmail.com] anywhere within this region and account for $10 \%$ to $15 \%$ of primary pediatric intracranial tumors. Until about two decades ago, tumors within the brainstem were considered inoperable due to the high morbidity

and mortality associated with surgery in this anatomical location. Experience over the last several decades has demonstrated that brainstem gliomas comprise a heterogeneous group of tumors, some of which with favorable long-term survival.

The brainstem is a highly complex structure, acting as a station where fibers from the cerebral cortex transit up- and downstream the neural axis. Furthermore, the fact that nuclei of the cranial nerves, neural networks and other vital structures lay compactedly arranged in such a tiny space represents a great challenge for the neurosurgeon to approach intrinsic brainstem lesions [1-3] (Figure 1).

From the 1980s, several authors started publishing series with expressive results, initiating a new era in brainstem tumor surgery. Postoperative care has also evolved since then, mainly regarding the prevention of respiratory complications and swallowing disorders. The approach to brainstem tumors during that initial era was rather aggressive, nevertheless, as a result it could be demonstrated that not all intrinsic lesions were malignant tumors $[2,5]$.

The development of neuroimaging allied to the refinement of surgical tools, such as improved magnification and visualization of the surgical field with more powerful surgical microscopes and sources of light, followed by the introduction of ultrasonic aspirator, anesthetic and intraoperative neurophysiological monitoring provided the neurosurgeon with conditions for a much safer surgical management of these tumors [6].

This paper aims to review brains steam tumors in children, from clinical presentation, imaging and treatment.

Journal homepage: www.sbnped.com.br 


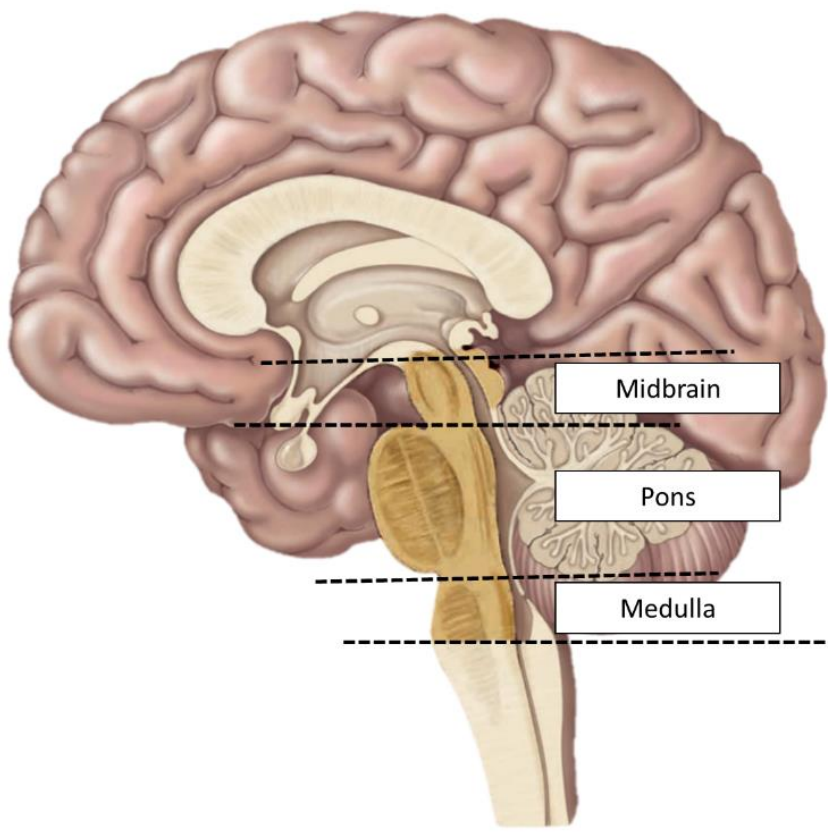

Figure 1 - Anatomical location of the brainstem and its classic subdivisions.

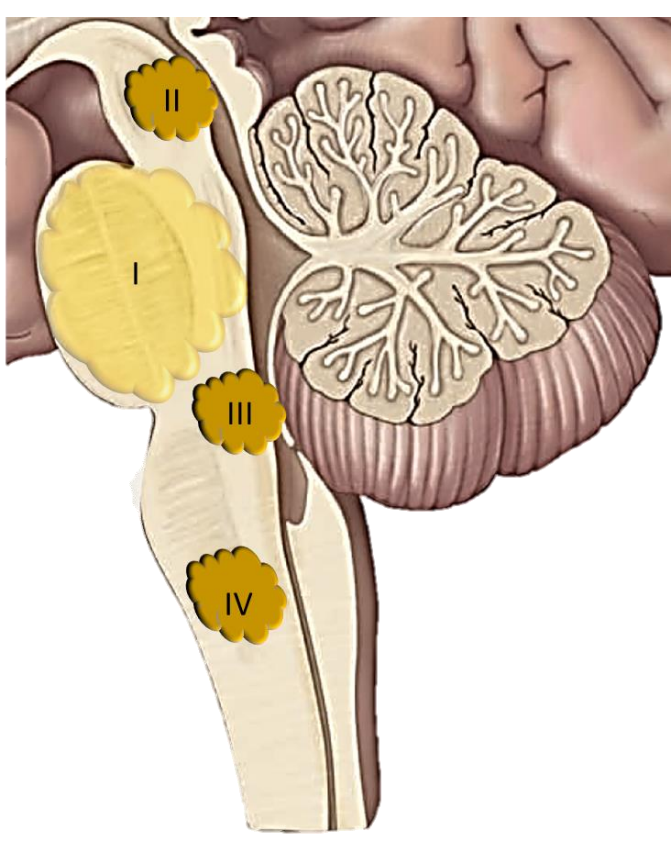

Figure 2 - Choux et al. classification for brain stem tumors.

\section{Clinical Presentation}

The average age of onset of BSG ranges from 5 to 10 years, with equal incidence among boys and girls. There are no recognized premalignant lesions, however a number of familial cancer syndromes have been associated with it, including neurofibromatosis type 1, Li-Fraumeni syndrome, Gorlin syndrome, tuberous sclerosis, among others.

The specific signs and symptoms of brainstem tumors are dependent on their anatomical location. Children with Diffuse Intrinsic Pontine Glioma (DIPGs) typically have a rapidly progressive course, usually with dysfunction of cranial nerves, pyramidal and cerebellar pathological signs. In contrast, the presentation of focal brainstem gliomas is more insidious with localizing signs such as isolated cranial nerve deficits and contralateral hemiparesis spanning months to years. Tumors of the cervicomedullary junction commonly present with lower cranial nerve palsy, pyramidal tract signs, ataxia, spinal cord dysfunction, and nystagmus. Signs and symptoms of hydrocephalus usually manifest later along with the progression of the disease, exception for tectal tumors, which may present with obstructive hydrocephalus due to its close relationship with the cerebral aqueduct [7-9].

\section{Imaging}

The radiological investigation of brainstem tumors has advanced a lot with the use of computed tomography (CT) and more recently magnetic resonance imaging (MRI). Tractography (DTI) has facilitated the planning of the best surgical approach for these lesions, showing that the fibers are often deviated and not infiltrated by the tumor.

One of the currently most used brain steam tumors classifications was published in 1999 by Choux et al. [1]. The authors subdivided brainstem tumors into four types based on radiological findings: type I - intrinsic and diffuse tumor; type II - intrinsic and focal tumor, which may be solid or cystic and located in one of the three anatomical subdivisions of the brain stem; type III - exophytic tumor, which may be lateral or dorsal; and type IV - cervico-medullary tumors (Figure 2). This is a valuable classification to have in mind, since it will serve as a reference guide towards the appropriate treatment strategy, helping the attending neuro-oncology team to select patients for clinical and/or surgical management.

In general, the focal and exophytic types are lowgrade gliomas and carry a much better prognosis than the diffuse high-grade glioma. DIPGs account for $60 \%$ to $80 \%$ of brainstem gliomas.

\footnotetext{
Diffuse tumors (Type I) - Diffuse Intrinsic Pontine Glioma (DIPG)

DIPGs are the most common brainstem tumors, accounting for approximately $60 \%$ to $80 \%$ of lesions [9]. Unfortunately, DIPGs are the most devastating brainstem tumor with a median survival of 9 months despite treatment with multiple therapeutic modalities. Children usually present with rapid onset and progression of a triad of symptoms (cranial nerve palsy, long tract and cerebellar signs). Hydrocephalus occurs in the advanced stages of this disease. These lesions have a characteristic appearance on MRI. They appear hypointense on T1-weighted and hyperintense on T2-weighted images with indistinct
} 
margins, reflecting their infiltrative nature. On midsagittal imaging, their confinement to the pons is better delineated. Gadolinium enhancement is variable and provides no additional prognostic information. Magnetic resonance spectroscopy (MRS) is an evolving imaging modality in brainstem tumors. DIPGs have increased metabolic ratios of choline to creatine and $\mathrm{N}$-acetylaspartate, which is useful in delineating DIPG from demyelination, dysmyelination of NF1, encephalitis, and radionecrosis [14-15]. (Figure 3)

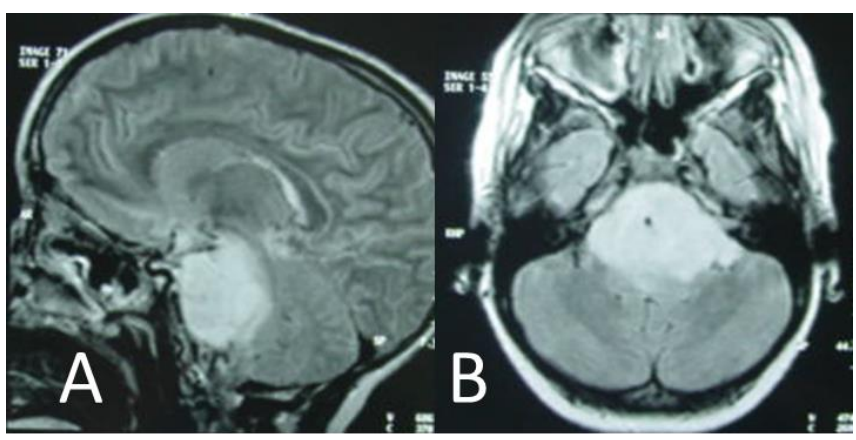

Figure 3 - Diffuse tumor (Type I) affecting the pons. (A) Sagittal Flair (B) Axial Flair

\section{Focal Brainstem Tumors (Type II)}

Tectal tumors are intrinsic lesions in the region of the mesencephalon, representing $5 \%$ of brainstem lesions and are typically WHO grade I or II. Given their close proximity to the cerebral aqueduct, tectal tumors may cause hydrocephalus, rapid deterioration, and death even when small sized. Although the vast majority of tectal gliomas exhibit an indolent benign course $(>85 \%)$, a subtype of this tumor may behave more aggressively. MRI may help to distinguish between them, as the more aggressive subtype is typically greater than $2 \mathrm{~cm}$ at presentation, may invade the adjacent tegmentum, thalamus and pons, and present post-gadolinium enhancement.

The most common benign intrinsic tectal tumors generally follow an indolent course. These are typically well-circumscribed nonenhancing low-grade astrocytomas, presenting clinically with signs and symptoms of intracranial hypertension due to obstructive hydrocephalus [15]. Other signs include gait disturbance, ataxia, Parinaud syndrome, and strabismus. There is still a debate regarding the best treatment strategy for nonsuspicious tectal lesions. The treatment of underlying obstructive hydrocephalus followed by watchful waiting may be the safest approach to these lesions. Nevertheless, some centers favor a more aggressive surgical approach given that $18 \%$ to $30 \%$ of tectal tumors eventually progress (16).

Hydrocephalus can be treated with a ventriculoperitoneal shunt or preferably via endoscopic third ventriculostomy (ETV), which has also demonstrated to be safe and effective. It is crucial to follow-up these patients closely, because of the risk of tumor progression and eventual ETV/Shunt failure, leading to serious complications (Figure 4) [17].

Aggressive subtypes of tectal tumors are typically larger on presentation compared to the indolent variety. It can be associated with neurofibromatosis and sometimes surgical resection followed by radiation therapy could be a treatment option.

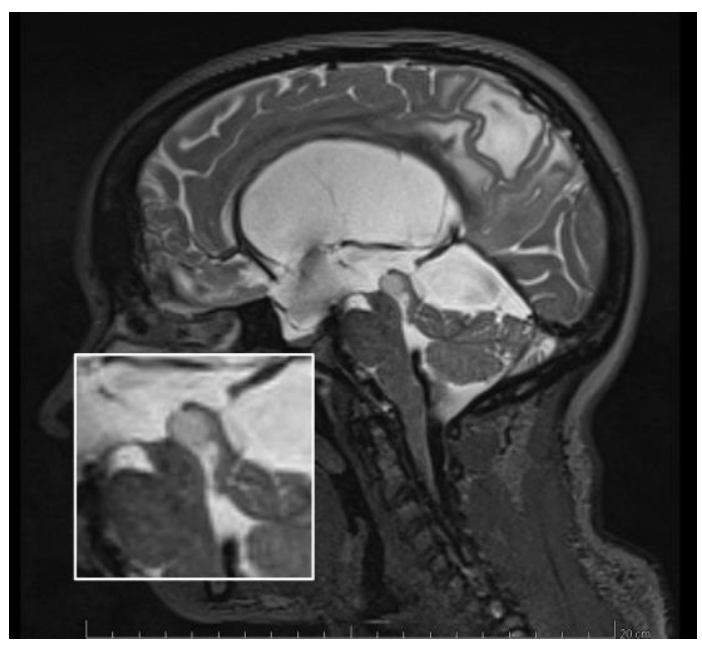

Figure 4 - Focal tumor (Type II) of the quadrangle lamina with hydrocephalus, submitted to VTE - Sagittal T2

Dorsally Exophytic Brainstem Tumors (Exophytic tumors) - (Type III)

The dorsally exophytic subtype accounts for approximately $10 \%$ to $20 \%$ of brainstem tumors. Children usually present with an insidious history of headaches, vomiting, ataxia, and cranial nerve dysfunction (usually sixth and seventh). Papilledema, torticollis, and long tract signs can be found on neurological examination. By definition, these tumors protrude into the fourth ventricle, but occasionally they can be dorsolaterally exophytic, projecting into the cerebello-pontine angle (CPA). The hypointense signal on $\mathrm{T} 1$ and the hyperintense signal on T2 will generally display consistent tumor edges reflecting its less infiltrative nature. Contrast enhancement is typical. These tumors are predominantly pilocytic astrocytomas with occasional grade 2 and 3 astrocytomas, or gangliogliomas, generally carrying a good prognosis. Dorsally exophytic tumors are the most surgically accessible of all brainstem gliomas. Similar to pilocytic astrocytomas elsewhere in the neural axis, the treatment of choice is microsurgical resection. (Figure 5).

\section{Cervicomedullary Tumors (Type IV)}

Children with cervicomedullary tumors commonly present with slowly progressive lower cranial nerve palsy, pyramidal tract signs, ataxia, spinal cord dysfunction, or nystagmus. Lower cranial nerve deficits may include dysphagia, nasal speech, nausea, vomiting, palate deviation, facial nerve palsy, head tilt, 
apnea, or irregular breathing patterns. The gradual onset of these symptoms reflects the slowly growing,

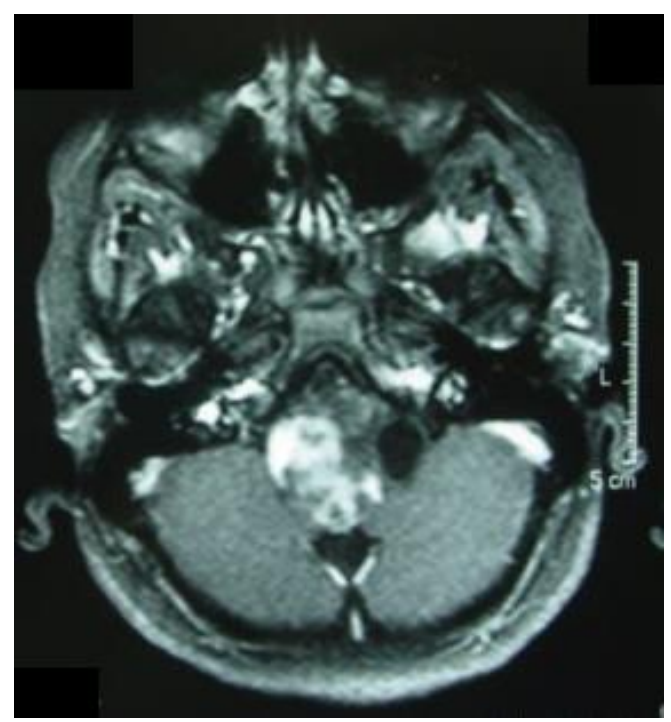

Figure 5 - Exophytic ganglioglioma (type III) bulbar - Contrast

relatively benign histological picture that is most commonly found, namely, the pilocytic astrocytoma.

These tumors typically extend from the caudal two thirds of the medulla to the rostral portion of the cervical spinal cord.

The prognosis is generally favorable following neurosurgical resection, given the low-grade nature of the majority of these lesions. An aggressive surgical resection is usually undertaken assumed that these tumors are more likely to have a defined surgical plane compared to other brainstem tumors. However, surgery in this location carries significant risks including quadriparesis, sleep apnea, cranial nerve palsy, proprioceptive deficits, and spasticity. Postoperative radiation therapy may be indicated, although most reference centers would opt for watchful-waiting until any evidence of recurrence. (Figure 6)
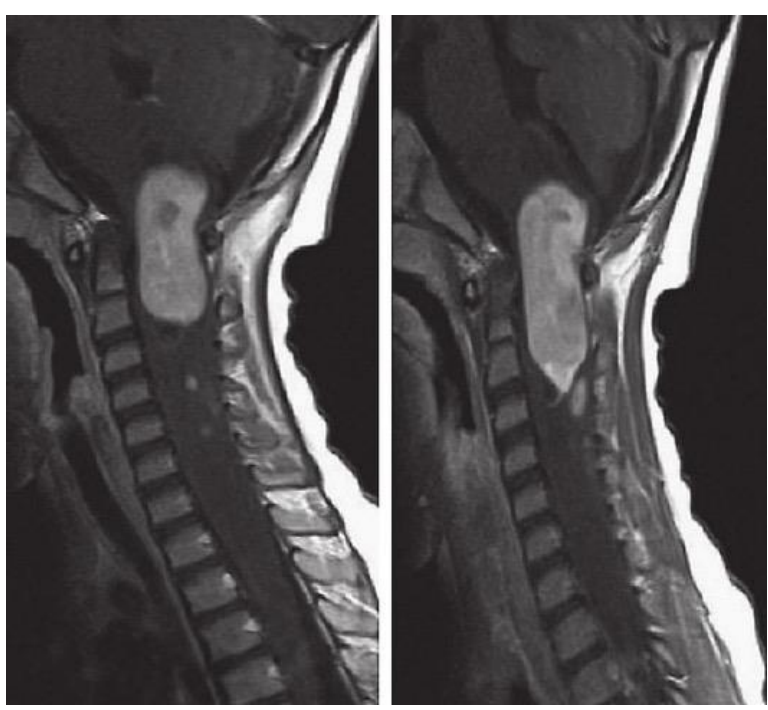

Figure 6- Pilocytic astrocytoma in the cervicomedullary transition (type IV) in a patient with type 1 neurofibromatosis.

\section{Differential diagnosis}

The main differential diagnosis of brainstem tumors in children are vascular malformations (e.g. cavernous angioma), ependymal cysts, parasitic diseases and demyelinating diseases[13].

\section{Treatment}

The decision-making regarding the best treatment strategy for brainstem tumors should be undertaken case-by-case, within a multidisciplinary team, and shared with patient's carers. Several factors may influence the approach of choice, such as the tumor location, risks involved, surgical routes and adjuvant treatments available (18).

\section{Diffuse Intrinsic Pontine Glioma (DIPG) - Type I}

Because the natural history and malignant course of DIPGs are well established, there is currently little clinical role for a diagnostic biopsy. A biopsy is reserved for indeterminate lesions on MRI, unusual presentations, or in the setting of a clinical study. Many authors have shown that biopsies can be accomplished with reasonable safety, with sufficient tissue for a histological diagnosis and complication rates ranging from $10 \%$ to $30 \%$.

The rationale is that nearly all DIPGs are highgrade astrocytomas, outcomes are poor regardless of pathological grade, treatment strategies do not hinge on tumor grade, and biopsy is associated with significant morbidity and mortality rates. However, if we are to gain new insight into the biological and genetic behavior of these tumors, in the hopes of designing targeted therapies, biopsy may become an important strategy in the future.

Pincus et al. [19], carrying out a retrospective study of 182 cases of stereotaxic biopsy, collected in thirteen series in the literature for DIPG found that the diagnosis of the tumor was verified in 75 to $100 \%$ of the cases. In $87 \%$ of the cases, the lesions were gliomas; in the remaining $13 \%$ the lesions were: primitive neuroectodermal tumors, neurocytomas, ependymomas and demyelinating lesions. Morbidity ranged from zero to $16 \%$ and mortality reached up to $5 \%$.

Roujeau et al. [20] published the results of stereotactic biopsy in 24 pediatric patients. There was no surgical mortality in that series. The authors' arguments for performing a stereotaxic biopsy were: the need for a sample to define treatment strategy and the need for material for molecular biology studies.

On the other hand, the sample obtained may be insufficient and may not provide an accurate diagnosis 
or promote erroneous diagnoses (heterogeneous tumor), in addition to the risk of biopsy [21].

\section{VENTRAL}

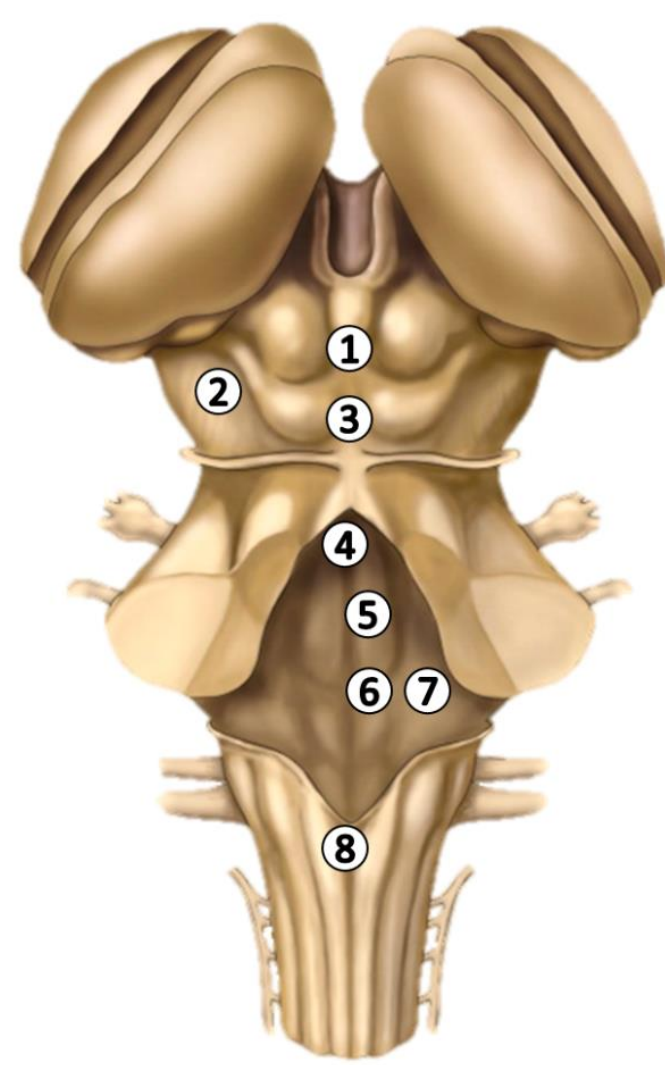

DORSAL

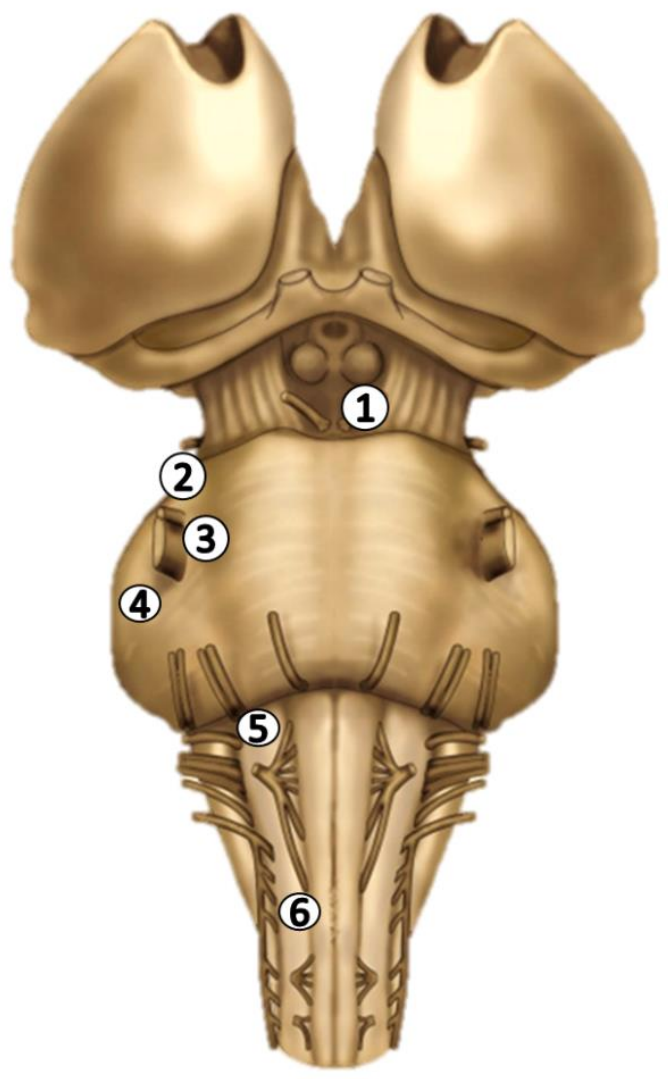

Figure 7 - "Safe" entry zones for surgical access to the brainstem. VENTRAL: 1 - Supracollicular zone. 2- Lateral mesencephalic sulcus. 3 Infracolicular zone. 4 - Median sulcus of 4th ventricle. 5 - Supracollicular paramedian . 6 - Infracollicular paramedian. 7 - Acoustic area. 8 - Dorsal sulcus of the medulla. DORSAL: 1 - Anterior mesencephalic zone. 2 - Supratrigeminal zone. 3 - Peritrigeminal zone. 4 - Side pons area. 5 - Olivary zone.6 - Anterolateral sulcus.

Thus, it can be concluded that biopsy would be indicated only in cases of non-characteristic images or in research centers, respecting study protocols and informed consent. Albright [22] argued that the biopsy should only be performed when there is a great doubt regarding the radiological aspect or when the lesion extends beyond the limits of the pons. In general, the treatment of this type of tumor is frustrating and the prognosis poor, where most children will not survive the first year following diagnosis. [23, 24].

Molecular biology studies indicated that mutations in histone (H3) and ACVR1 [25], H3F3A / K27M and G34R / V [26] may be potential therapeutic targets [27].

\section{Focal Brainstem Tumors - Type II}

The selection of appropriate surgical approach and route to the brainstem has to be carefully evaluated preoperatively. The different radiological sequences allow to accurately assess the best trajectory. Lesions presenting extension towards the cerebellopontine angle but actually arising within the brainstem can be approached through a classic suboccipital / telovellar approach through the fourth ventricle or directly through a retrosigmoid approach, in children. The surgical position may vary according to the surgeon's preferences and experience.

Tumors located in the midbrain require a different route. An interfornicial transcallosal approach or even, an endoscopic/microscopic approach through the foramen of Monro may be chosen for tumors growing towards the most anterior portion of the brainstem [28].

The lesions located in the posterior portion of the midbrain or even in the quadrigeminal plate, reaching the third ventricle, can be removed through a supracerebellar infratentorial route.

In some situations, the combined pre- and subtemporal supratentorial approaches can be very useful for the management of these mesencephalic lesions [29]. 


\section{Surgical rationale}

Surgery may be indicated for focal tumors, except for those located within the quadrigeminal plate. The survival for these tumors is around $80 \%$ in five years and $70 \%$ in 10 years, therefore, surgical treatment may be of advantage [9].

Brainstem tumor surgery requires experience from the neurosurgical team, in addition to equipment and the use of neurophysiological monitoring. Whenever possible, the cranial nerves as well as the somatosensory and motor evoked potentials should be used in order to reduce surgery-related morbidity.

Although the brainstem is an extremely complex structure, some safe routes and entry-zones provide access for surgery with low morbidity [16, 30] (Figure 7).

When there is no clear lesion on the surface of the brainstem, the decision to approach the brainstem should be done by a midline incision, between the upper and lower colliculi. The brainstem can be approached above the facial colliculus, and even below the medullary striae (9). However, when the tumor dislocates anatomical structures, it may be challenging to accurately identify the midline. In this situation, intraoperative monitoring is essential.

The use of electrical coagulation in the brain stem must be avoided, and, when absolutely necessary, always concomitant with irrigation, as the heat released by the bipolar can cause permanent damage.

The ultrasonic aspirator is a very important resource for the removal of brainstem tumors, avoiding traction and, consequently, autonomic disorders. It is important to use a thin and delicate ultrasonic aspirator at low power settings and gently so as not to cause bleeding during dissection. During brain tumor removal, continuous anesthetic monitoring is essential and good communication between surgeon, neurophysiologist and anesthetic team is required.

If any autonomic changes occur, such as tachycardia, high blood pressure and bradycardia, surgery must be stopped until improvement of physiological signs to normal ranges.

Irrigation with warm saline solution is also important to keep the surgical field clean, promote hemostasis and facilitate neurophysiological monitoring.

Total surgical resection should be aimed whenever possible with maximum safety and preservation of function. Thus, the neurosurgeon must be prepared to interrupt surgery if needed, and plan a second intervention when appropriate.

Intrinsic medullary tumors tend to have good survival rates and stable progression after surgery; however, the perioperative risks of ventilation, tracheostomy, and gastrostomy were high in some series [9]. Patients with cervicomedullary tumors can achieve favorable results with surgery, with 4- and 5year survival rates of $72 \%$ to $100 \%$. In cervicomedullary tumors, the degree of preoperative morbidity predicts postoperative function.

The general principles for surgical debulking of BSGs include (1) identifying normal anatomy; (2) identifying the most direct route to the tumor, either by exploring tumor cysts or locating pial surfaces with discoloration for tumor bulge; and (3) debulking the center of the lesion prior to dissecting tumor margins.

Neurophysiological monitoring such as brainstem auditory evoked responses, somatosensory evoked potentials, electromyography and motor evoked potentials, and neuronavigation are useful adjuncts in minimizing injury to the patient.

\section{Complications}

Complications can be classified as acute, postoperative and permanent.

- Acute complications (intraoperative) - vascular lesions (deep draining veins, arteries), cerebellar edema, autonomic disorders (bradycardia, hypertension), death;

- Postoperative complications - hydrocephalus, CSF fistula, drowsiness, coma, nerve injuries or cranial nuclei (facial paralysis, diplopia, paralysis, extrinsic eye movement, swallowing, dysphonia, among others), hemiparesis, tetraparesis, mutism, alteration of balance, death, among others;

- Permanent complications - usually related to injuries to cranial nerves / nuclei; motor deficit, balance changes.

\section{Conclusion}

Despite improvements in understanding the biology and advances in treatment strategies (particularly radio- and chemotherapy), brainstem tumors continue to present a therapeutic challenge for neurosurgeons. Poor patient outcomes, especially for DIPGs, are still commonplace. Surgical advancements in techniques, neurophysiological monitoring and neuroimaging have allowed many patients with focal and dorsally exophytic brainstem tumors to experience a prolonged tumor-free survival. Not all brainstem tumors have an unfavorable prognosis. Subsets of focal brainstem gliomas are associated with long-term survival. Diffuse intrinsic pontine gliomas account for $60 \%$ to $80 \%$ of brainstem gliomas, with an average survival of 9 months. NF1 patients have brainstem tumors with a more favorable survival time.

\section{Disclosure Statement}

The authors have no conflicts of interest to declare.

\section{Funding statement}

None. 


\section{References}

1. Choux M, Lena G. Brainstem tumors. In: Choux M, Walker M, DiRocco C, editors. Pediatrics Neurosurgery. Churchill Livingstone (London)1999. p. 471-91.

2. Morcos JJ, Haines SJ. History of brain stem surgery. Neurosurgery clinics of North America. 1993;4(3):357-65.

3. Martirosyan NL, Carotenuto A, Patel AA, Preul MC. History of Brainstem Surgery. In: Spetzler R, Kalani M, Lawton M, editors. Surgery of the Brainstem. New York: Thieme Publishers 2020.

4. Pool JL. Gliomas in the Region of the Brain Stem. 1968;29(2):164.

5. Grimm SA, Chamberlain MC. Brainstem glioma: a review. Current neurology and neuroscience reports. 2013;13(5):346.

6. Cavalheiro S, Yagmurlu K, da Costa MDS, Nicácio JM, Rodrigues TP, Chaddad-Neto F, et al. Surgical approaches for brainstem tumors in pediatric patients. Child's Nervous System. 2015;31(10):1815-40.

7. Cavalheiro S, Inácio JM, Faquini I. Tumores do Tronco Encefálico. In: Siqueira MG, editor. Tratado de Neurocirurgia 2015. p. 1683-92

8. Maher CO, Raffel C. Neurosurgical treatment of brain tumors in children. Pediatric clinics of North America. 2004;51(2):327-57.

9. Cavalheiro S, Loduca R. Tumores do tronco encefálico. In: de Oliveira RS, Machado HR, editors. Neurocirurgia Pediátrica: fundamentos e estratégias. Rio de Janeiro-RJ: Di Livros; 2009. p. 240-6.

10. Albright L, Pollack I. Brainstem gliomas. In: Winn H, editor. Youmans Neurological Surgery. Philadelphia: Saunders; 2003.

11. Epstein F, McCleary EL. Intrinsic brain-stem tumors of childhood: surgical indications. Journal of neurosurgery. 1986;64(1):11-5.

12. Konovalov A, Gorelyshev S, Khuhlaeva E. Surgery of diencephalic and brain stem tumors. In: Schmidek H, Sweet W, editors. Operative neurosurgery techniques: indications, methods, and results. WB Saunders Company Philadelphia; 1995. p. 765-82.

13. Volpon Santos M, Furlanetti LL, Jeanne Bezerra MD, Santos de Oliveira R. Epidermoid cyst mimicking an intrinsic brainstem tumor. Neurocirugia (Asturias, Spain).

2013;24(3):135-8.

14. Osborn AG. Astrocytomas. In: Osborn AG, Hedlund GL, Salzman KL, editors. Osborn's brain. 2 ed. Salt Lake City, UT: Elsevier; 2018. p. 509-52.

15. Puget S, Boddaert N, Veillard AS, Garnett M, Miquel C, Andreiuolo F, et al. Neuropathological and neuroradiological spectrum of pediatric malignant gliomas: correlation with outcome. Neurosurgery. 2011;69(1):215-24. 16. Cavalheiro S, Yagmurlu K, da Costa MD, Nicácio JM, Rodrigues TP, Chaddad-Neto F, et al. Surgical approaches for brainstem tumors in pediatric patients. Child's nervous system : ChNS : official journal of the International Society for Pediatric Neurosurgery. 2015;31(10):1815-40.

17. Puget S, Crimmins DW, Garnett MR, Grill J, Oliveira $\mathrm{R}$, Boddaert N, et al. Thalamic tumors in children: a reappraisal. Journal of neurosurgery. 2007;106(5 Suppl):35462.

18. Faulkner H, Arnaout O, Hoshide R, Young IM, Yeung JT, Sughrue ME, et al. The Surgical Resection of Brainstem Glioma: Outcomes and Prognostic Factors. World neurosurgery. 2020.

19. Pincus DW, Richter EO, Yachnis AT, Bennett J, Bhatti MT, Smith A. Brainstem stereotactic biopsy sampling in children. Journal of neurosurgery. 2006;104(2 Suppl):108-14. 20. Roujeau T, Machado G, Garnett MR, Miquel C, Puget S, Geoerger B, et al. Stereotactic biopsy of diffuse pontine lesions in children. Journal of neurosurgery. 2007;107(1 Suppl):1-4.

21. Scott RM. Pontine Tumors - Letter 1. 2008;1(5):423.

22. Albright AL. Diffuse brainstem tumors: when is a biopsy necessary? Pediatric neurosurgery. 1996;24(5):252-5. 23. Recinos PF, Sciubba DM, Jallo GI. Brainstem tumors: where are we today? Pediatric neurosurgery. 2007;43(3):192201.

24. Reithmeier T, Lopez WO, Spehl TS, Nguyen T, Mader I, Nikkhah G, et al. Bevacizumab as salvage therapy for progressive brain stem gliomas. Clinical neurology and neurosurgery. 2013;115(2):165-9.

25. Green AL, Kieran MW. Pediatric brainstem gliomas: new understanding leads to potential new treatments for two very different tumors. Current oncology reports.

2015;17(3):436.

26. Oliveira VF, De Sousa GR, Dos Santos AC, Saggioro FP, Machado HR, de Oliveira RS, et al. Evaluating H3F3A $\mathrm{K} 27 \mathrm{M}$ and G34R/V somatic mutations in a cohort of pediatric brain tumors of different and rare histologies. Child's nervous system : ChNS : official journal of the International Society for Pediatric Neurosurgery. 2020. 27. Long W, Yi Y, Chen S, Cao Q, Zhao W, Liu Q. Potential New Therapies for Pediatric Diffuse Intrinsic Pontine Glioma. Frontiers in pharmacology. 2017;8:495. 28. Augusto LP, Ballestero MFM, de Oliveira RS. Interhemispheric transcallosal transchoroidal approach for resection of a mesencephalic lesion. Archives of Pediatric Neurosurgery. 2019;1(1(September-December)):28. 29. de Oliveira RS, Maia WS, Santos MV, Camara RL. Combined pre- and subtemporal transtentorial approach for epidermoid cysts of the cerebellopontine angle. Child's nervous system : ChNS : official journal of the International Society for Pediatric Neurosurgery. 2012;28(12):2137-42. 30. Cavalcanti DD, Preul MC, Kalani MY, Spetzler RF. Microsurgical anatomy of safe entry zones to the brainstem. Journal of neurosurgery. 2016;124(5):1359-76. 